\title{
Identification of biofilm producing bacterial species causing dental caries and their sensitivity to zoocin A and clove hydroalcoholic extract
}

\author{
Haniyeh Khalili ${ }^{1}$, Rouhollah Sisakhtpour ${ }^{1}$, Sedigheh Abdollahzadeh ${ }^{2 *}$ \\ ${ }^{1}$ Department of Bacteriology, Faculty of Medical Sciences, Tarbiat Modares University, Tehran, Iran \\ ${ }^{2}$ Department of Genetics, Shahrekord University of Medical Sciences, Shahrekord, Iran
}

\author{
*Correspondence to \\ Sedigheh Abdollahzadeh, \\ Email: bacteriology94@gmail.com \\ Received 29 January 2018 \\ Accepted 20 April 2018 \\ Published Online 5 May 2018
}

Keywords: Dental caries, Biofilms, Zoocin A, Cloves

Citation: Khalili H, Sisakhtpour R, Abdollahzadeh $\mathrm{S}$. Identification of biofilm producing bacterial species causing dental caries and their sensitivity to zoocin $\mathrm{A}$ and clove hydroalcoholic extract. Immunopathol Persa. 2018;4(2):e27. DOI:10.15171/ ipp.2018.27.

\begin{abstract}
Introduction: Dental caries is an infectious disease mostly associated with streptococcal species, especially Streptococcus mutans, as the main etiologic agent. Zoocin A protein is extracted as a recombinant protein from Streptococcus zooepidemicus and Escherichia coli with high effect on S. mutans. Clove extract has also been shown to have antimicrobial effects on several bacterial species, such as $S$. mutans.

Objectives: The purpose of this study was to isolate and identify common bacteria causing dental caries and their susceptibility to zoocin A and clove extract.

Patients and Methods: This study was performed on 133 dental caries samples in Tehran province. The isolates were identified by phenotypic and molecular methods. Molecular identification was performed by $16 \mathrm{~S}$ rRNA gene sequence. The effects of antibiotics, zoocin A and clove extract were studied.

Results: There was no significant difference between men and women regarding dental caries $(P=0.422)$, but age group of $16-30$ years were the most common $(P=0.002)$. Dominant biofilm forming bacteria identified with 16srRNA analysis including S. mutans, S. mitis, S. sanguinis, S. gordonii, Lactobacillus gasseri, Jemela hemolysin, and Granulicatella adiacens. Zoocin A exhibited a strong inhibitory effect with minimum inhibitory concentration (MIC) range of 2-4 $\mu \mathrm{g} / \mathrm{mL} \mathrm{mostly}$ against S. mutans. MIC of hydroalcoholic extract of clove was $8-16 \mu \mathrm{g} / \mathrm{mL}$.

Conclusion: Streptococcal species were predominant causes of tooth decay. Zoocin A and clove extract showed antimicrobial activity even on antibiotic-resistant and biofilm-forming species causing tooth decay.
\end{abstract}

\section{Introduction}

Streptococcus mutans (including S. mutans and S. sobrinus sub-species) are often involved in tooth decay. However, dental caries lesions contain a wide range of bacterial species, most of which have not yet been cultured, and yet the bouts of each bacterial species have not yet been determined individually in triggering tooth decay. Other bacteria other than $S$. mutans, including $S$. mitis, $S$. salivarius and $S$. anginosus, along with Enterococcus faecalis, Actinomyces naeslundii and a number of other lactobacilli causing tooth decay $(1,2)$. However, $10 \%$ of people with caries do not have significant levels of $S$. mutans, indicating the presence of other oral species effective in making caries. Likewise, $S$. mutans is significantly associated with decay

\section{Key point}

Zoocin A and clove extract have antimicrobial activity even on antibiotic-resistant and biofilmforming species causing tooth decay.

lesions and isolates from decay lesions may be more acidic than isolates from healthy teeth $(3,4)$.

Streptococcus mutans is a hetero-fermentative (multi-fermentable) bacterium, which allows it to produce the final product of the acid mixture, and has an advantage for growth in bacterial plaques. This leads to extensive acid production following digestion of carbohydrates, and thus reduces the $\mathrm{pH}$ of the dental plaque. Sucrose as a vital factor in $S$. mutans virulence is very important in dental caries and formation of biofilms (5).

Copyright (C) 2018 The Author(s); Published by Nickan Research Institute. This is an open-access article distributed under the terms of the Creative Commons Attribution License (http://creativecommons.org/licenses/by/4.0), which permits unrestricted use, distribution, and reproduction in any medium, provided the original work is properly cited. 
Approximately 2.4 billion people (36\% of the world's population) have permanent teeth rotting. The disease affects 620 million children or $9 \%$ of the world's population (6). The disease is prevalent in Latin America, the Middle East, and South Asia, with the lowest prevalence in China. In the United States, dental caries is the most common chronic disease in childhood and is five times more common than asthma. In children, the main pathological cause is the loss of teeth. Between $29 \%$ and $59 \%$ of adults over 5 years of age suffer from dental caries (7).

Zoocin A specifically attacks the cell wall of a number of similar Streptococcus species, including the Streptococcus A group causing sore throats and tooth decay $(8,9)$. Zoocin A suppresses the growth of $S$. mutans in a three-plate model, which the onset of suppression of these species begins very quickly.

Caryophyllus aromaticus is a scientific name for the cluster tree, which is native to the Molok Islands and eastern Indonesia, and is found in Tanzania, Madagascar, Brazil, and temperate and tropical regions. Due to its particular beauty, it is grown today as the ornament in the most part of the world.

The most important properties of clove are the antiviral effects of its aqueous extracts in skin lesions due to the herpes virus. Eugenol is a significant therapeutic effect of its hepatic protection. Clinical research has been carried out to evaluate the therapeutic benefits of clove in the treatment of pain and inflammation of oral and dental diseases, as well as to reduce the headache of patients.

\section{Objectives}

The purpose of this study was to isolate and identify common bacteria causing dental caries and their susceptibility to zoocin A and clove extract.

\section{Patients and Methods}

A total of 133 samples were randomly selected from patients referred to dental clinics in eight Tehran townships. Patients completed questionnaires prepared by the research organization. This questionnaire was based on gender, oral health and dental care based on the Likert scale and IDC-M10 classification. To isolate common oral bacteria, $5 \mathrm{ml}$ of sterile phosphate saline buffer was first placed in each of the sterile test tubes and then, using sterilized swabs from the buccal surface and the location of the caries of the target teeth, the samples were taken. Samples were stored in a portable refrigerator $\left(\sim 4^{\circ} \mathrm{C}\right)$ and transferred to the laboratory (the transfer time was typically 3 hours). Samples were stored at $80^{\circ} \mathrm{C}$ until further examination.

\section{Isolation of common bacteria causing tooth decay}

To isolate the bacteria, samples were vortexed for 30 seconds before the bacteria were isolated from the swab and inserted into the phosphate saline buffer solution. Then $100 \mu \mathrm{L}$ of the suspension was removed and diluted in saline buffer. Then, $20 \mu \mathrm{L}$ of each diluted suspension was put on a heart and brain infusion (BHI) culture medium. The plates were then incubated at $37^{\circ} \mathrm{C}$ for 48 hours. Plates were then placed in aerobic and anaerobic conditions (in anaerobic jar). After 48 hours, the colonies were isolated and cultured on Coliform Agar with 5\% dehydrated sheep blood, Tadd Hewitt Agar (THA), and Luria-Bertani (LB) medium (10).

\section{Biofilm formation assay}

A quantitative study on the biofilm formation was performed by tube staining and microtiter tissue plate assay. One $\mathrm{ml}$ of $\mathrm{LB}$ broth was mixed with $100 \mu \mathrm{L}$ of secondary culture colonies and incubated at $37^{\circ} \mathrm{C}$ at 24 , 48 and 72 hours. The contents of the tubes were then poured out and dried with phosphate saline buffer (PBS). The pipes were stained using $0.1 \%$ crystal violet. The tubes were then washed again with deionized water (ionized) to remove excess dye. The presence of violet-colored spots on the walls of the pipes indicated the formation of biofilms (11).

In the microtiter-plate assay, the colonies were cultured on BHI, Colombia agar and THA media. Then, a colony was dissolved and diluted to opacity equal to half McFarland and in saline and $200 \mu \mathrm{L}$ inoculated into microplate wells in triplicate. All three colony-free culture media were used as negative control. The plates were incubated for 24,48 , 72 hours at $37^{\circ} \mathrm{C}$ and biofilm formation was investigated. Thereafter, the microtiter plates were reversed and gradually thawed out of the contents. To remove planktonic bacteria, the tubes were washed with phosphate saline buffer and were stained with $0.1 \%(\mathrm{w} / \mathrm{v})$ crystal violet solution and washed again and dried and suspended in 95\% ethanol. The opacity density (OD) of the tubes was read at $570 \mathrm{~nm}$. This visual density was considered as a biofilm adhesion index in each well. This test was repeated three times and its average was considered as an OD score (11).

\section{DNA extraction}

In order to extract DNA, the isolates were cultured in $\mathrm{LB}$ medium and incubated at $37^{\circ} \mathrm{C}$ for 24 hours. DNA extraction was performed as followings:

Firstly, $1.5 \mathrm{~mL}$ of suspended culture was inoculated into sterile microtubes and centrifuged at $7500 \mathrm{rpm}$ for 5 minutes to precipitate the bacteria. Then, the supernatant was discarded to obtain bacterial pellet. This was continued until bacterial sedimentation reached $100 \mu \mathrm{g}$ (12).

\section{Effect of zoocin A on the isolates}

To evaluate the inhibitory effect of recombinant zoocin A protein on the growth of the isolates, they were diluted and immersed in the MHA medium. Then, zoocin A antibacterial activity was investigated using two well and disk methods. After preparation of the agar, wells with 6 $\mathrm{mm}$ diameter and a depth of $3 \mathrm{~mm}$ were created using a tip of sterile pipettes. After each isolate was lawn, $20 \mu \mathrm{L}(2 \mu \mathrm{g})$ of zoocin A (Sigma-Aldrich, CAS \# L5027z) solution was poured into each well. The wells were then covered with a 
drop of Soft Agar. Finally, the plates were heated to $30^{\circ} \mathrm{C}$ for 24 to 48 hours. The inhibitory activity on the strains was measured and recorded on the basis of the diameter of the non-growth zone (in millimeters) using a caliper. Distilled water was used as a negative control.

In the disc method, after preparing the culture medium and isolates culture, the sterile blank disks were inoculated with zoocin A solution and incubated for 24 hours at $30^{\circ} \mathrm{C}$. On average, each disk was impregnated with $2-3 \mu \mathrm{g}$ of zoocin A. The ability to inhibit bacterial growth by discs was investigated based on the diameter of the inhibition zone. Tetracycline, vancomycin and erythromycin antibiotics were used as controls.

\section{The inhibitory effect of hydroalcoholic extract of clove on isolates}

Firstly, $20 \mathrm{~g}$ of clove powder was added to $50 \mathrm{~mL}$ of $50 \%$ ethanol and poured into a flask according to the method described previously (13). The soaked sample was tidied and closed with aluminum foil for 72 hours. The content of the flask was then filtered with Watchman paper No. 1 filter paper and placed in a soxhlet device. The solution was concentrated using evaporation. The resulting liquid was mixed with $200 \mathrm{~mL}$ distilled water and washed for 12 hours. Then, it was condensed with rotary incense at $40^{\circ} \mathrm{C}$. The condensed extract was stored in a glass bottle and stored at $4^{\circ} \mathrm{C}$ until use. The hydroalcoholic extract of clove was prepared.

To determine the inhibitory effect of hydroalcoholic extract of cloves on isolates, wells with a diameter of $6 \mathrm{~mm}$ and a depth of $3 \mathrm{~mm}$ were created using a tip of sterile pipettes. Then, in each well, $200 \mu \mathrm{L}$ of clove extract was inoculated using a sampler. The prepared plates were incubated at $30^{\circ} \mathrm{C}$ for 24 hours and inhibitory effect of the clove extract was observed by measuring the diameter of inhibitory zone.

\section{The minimum inhibitory concentration of zoocin A and clove extract}

In order to determine the minimum inhibitory concentration (MIC), various concentrations of zoocin A and different dilutions of hydroalcoholic clove extract were prepared in each well in the diffusion method. For this purpose, the isolates were cultured on the MHA medium, and then, $200 \mu \mathrm{L}$ of different dilutions of clove extract and various concentrations of zoocin A were poured into wells. After 24 hours of incubation, the inhibited growth zone was measured and recorded.

\section{Ethical issues}

The research followed the tenets of the Declaration of Helsinki. Before the study, written informed consent was obtained from all patients who participated in the study. Permission of the ethical review committee of Shahrekord University of Medical Sciences was obtained prior to execution of the study. All information about individuals was coded and kept confidential by the university.

\section{Statistical analysis}

The descriptive data collected in this study included the individual characteristics of the research units using IBM SPSS software version 20 was analyzed and reported as tables and charts. Statistical tests included $t$ test and ANOVA considering 95\% CI.

\section{Results}

\section{Patients' demographic data}

Most of the specimens from dental caries were from males, representing $55.4 \%(\mathrm{n}=72)$. In addition, most of the samples were among individuals in the age group of 16-30 years old.

Three quarts of samples had good or proper oral health score (score 5 or more) and the highest frequency was 8 (21.5\%) according to 0 - 10 scale range.

In this study, the degree of decay of teeth was categorized according to the IDC-M10 classification from World Health Organization Diseases Classification Subclasses, Edition 10 (ICD 10). According to this category, if the tooth decay is the main problem of the patient and is not due to illness and other complaints, the main complaint is considered and placed on the floor K02.1.

\section{Detection of 16s rRNA gene sequence}

Purified sequences of $16 \mathrm{~s}$ rRNA amplified by PCR were arranged using the ABI Prism cycle sequencing kit. The sequencing reactions were performed on the sequence of the ABI 3100 DNA strain. The DNA sequences were identified by the sequences in the human oral microbial database (HOMD) and compared through the site's software. Sequences that were less than $90 \%$ similarity with the sequence contained in the database were set aside. After sequencing of $16 \mathrm{~S}$ rRNA genes, identification of strains and phylogenic analysis was performed by comparing sequences with the HOMD database. Using the software, the database identified 114 classes that belonged to five different bacterial sequences. A total of 286 sequences were found to yield good and reliable results. The members of 5 various dynasties were Firmicutes, Actinobacteria, Fusobacteria, Bacteroides, and Proteobacteria. The distribution of the colonies was different in each of the samples, but on average 16S rRNA results showed that the members of the Firmicutes bacteria phylum dominated in all specimens. The similarity of bacteria in this range varied from $50 \%$ to $76 \%$ depending on the age of the specimens and $81 \%$ according to the health and oral health of the specimens. In addition to the Firmicutes phylum, only members of the Actinobacteria were found in all specimens, varying between $18 \%$ and $29 \%$ depending on the age and oral health status. Proteobacteria formed only $5 \%$ of the clones in the specimens. The members of the Fusobacteria, Prevotella and Bacteroides were found only in one sample. Totally, the microflora of dental caries included the Firmicutes and Actinobacteria with $81 \%$ and $19 \%$, respectively. 


\section{Biofilm formation}

Among the total 133 isolates, six were strong biofilm producers including S. mutans, S. mitis, S. sanguis, Lactobacillus gasseri, Jemela hemolysin and Granulicatella adiacens.

\section{Antibiotic susceptibility test}

All isolates were susceptible to the discs but resistant to vancomycin evaluated with the WHONET software.

Effect of zoocin $A$ and cloves on adopted biofilmproducing species

The results of zoocin A effect on six biofilm producing common oral bacterial species exhibited that the highest effect conferred on the Streptococcus spp. The MIC of zoocin A and cloves was significantly lower than control antibiotics (vancomycin, erythromycin and tetracycline).

\section{Discussion}

In the current study, $54 \%$ of the subjects were males and $45 \%$ were females $(P=0.422)$ and the age group of 16 to 30 years was predominant $(P=0.002)$, which may be the lack of cultural education at an earlier age or lack of attention to health due to lower income in this age range. In the 10-point Likert scale of oral health condition, each sample had at least one rotten tooth; three quarks had well or high oral health (score 5 or more) and the highest frequency was 8 (21.5\%). The majority of patients (59\%) had not filled up their teeth and prosthetic. In the present study, the 16S rRNA gene sequence was compared to those sequences in the human oral microbial database (HOMD) and those sequences with more than 90\% similarity included Actinobacteria, Fusobacteria, Bacteroides, and Proteobacteria. The bacterial species included S. mitis, S. mutans, S. sanguinis, S. gordonii, S. salivarius, L. gasseri and $L$. ines, G. adiacens. The results of sequencing in this study were comparable with results of Chandrabhan et al (14). In the initial biochemical tests and 20-strip API kit the identified bacteria included $S$. mitis, S. sanguinis, $S$. mutans, L. gasseri, J. hemolysin, and G. adiacens. Kim and Lee used the system and detected S. mutans, S. gordonii, S. mitis, S. oralis and S. sanguinis (15).

Over 700 species of bacteria have been identified in the oral cavity. In studies conducted using the DNA sequencing, the number of phylotypes in the oral cavity is more than 19000 . In one person, the number of bacterial species present in the oral cavity may be much less than these numbers, but these bacteria form a composite and complex biofilm $(16,17)$. The oral biofilm is a structured, three-dimensional community of bacteria attached to the oral surfaces, which are found to be considerably on enamel (18).

Those major bacteria contributing to the early stages of biofilm formation mainly include Streptococci, especially S. mitis and S. mutans, along with S. gordonii and S. anginosus and to a lesser extent Actinomyces spp, Eikenla, Neisseria, Haemophilus, prevotella, Propionibacterium and
Vionella spp $(18,19)$.

Chandrabhan et al determined 39 decay causing isolates from plaque and tooth decay and 13 strains had acidic properties. They consider the acidification and tolerance of acidic conditions to cause tooth decay. In sensitivity testing to common antibiotics, acidic bacteria exhibited higher resistance to antibiotics (14).

The results of determining the biofilm formation potential showed that six isolates were strong biofilm producers named from CF1 to CF6 all being gram-positive species. The results showed that zoocin A is highly effective on $S$. mutants and also inhibited $S$. gordonii, and demonstrated weaker effects on other species (MIC; $2-4 \mu \mathrm{g} / \mathrm{mL}$ ). These results are consistent with the findings of Lester and Simmonds (20), McLeod and Simmonds (21). Totally, zoocin A and cloves conferred high effect on six strong biofilm producer isolates including S. mutans, S. mitis, S. sanguis, L. gasseri, J. hemolysin and G. adiacens. The antimicrobial effect of clove extract on isolates showed that it conferred the highest effect $S$. mutans with a growth inhibition zone of $16.5 \mathrm{~mm}$, but had less effect on other bacteria species. The results of this study are consistent with those from previous studies conducted by Sergio et al (22), Chaiya et al (23) and Alitonou et al (24).

\section{Conclusion}

Streptococcus mitis and S. mutans were the most common species in dental caries in Tehran province. The zoocin A had a high inhibitory effect on these isolates (MIC=2$4 \mathrm{ug} / \mathrm{mL}$ ). Clove hydroalcoholic extract also conferred inhibitory effect. Therefore, these compounds can be used as alternatives to eradicate biofilms in oral cavity. In this study, the effect of selected antibiotics on isolates was investigated by WHONET software and it was found that isolates are sensitive to erythromycin $(\mathrm{MIC}=280)$ and tetracycline $(\mathrm{MIC}=146)$ but resistant to vancomycin. Several preventive options include the use of new antimicrobial agents, the use of artificial sweeteners instead of dietary sucrose, the replacement of cariogenic bacteria with non-infectious species, and vaccination.

\section{Limitations of the study}

The limitations of the study were low number of samples for study and lack of gene expression on the bacterial adhesion genes.

\section{Authors' contribution}

HK and RS conceived the study and collected the data from study participants. SA analyzed the data and drafted the final manuscript; all authors read, revised, and approved the final manuscript.

\section{Conflicts of interest}

There were no points of conflicts.

Ethical considerations

Ethical issues (including plagiarism, misconduct, 
data fabrication, falsification, double publication or submission, redundancy) have been completely observed by the authors.

\section{Funding/Support}

This study was supported by Shahrekord University of Medical Sciences, Shahrekord, Iran.

\section{References}

1. Kleinberg I. A mixed-bacteria ecological approach to understanding the role of the oral bacteria in dental caries causation: an alternative to Streptococcus mutans and the specific-plaque hypothesis. Critic Rev Oral Biol Med. 2002;13:108-25. dio: 10.1177/154411130201300202.

2. Van Houte J, Lopman J, Kent R. The predominant cultivable flora of sound and carious human root surfaces. I Dent Res. 1994;73:1727-34. doi: 10.1177/00220345940730110801.

3. Suman E, Devrari JC, Umesh U, Paul S, Suprabha B, Kotian SM. Effect of fluoride and chlorhexidine varnish on biofilm formation of Streptococcus mutans. Int Res J Biol Sci. 2015;4:4-7.

4. Simón-Soro A, Mira A. Solving the etiology of dental caries. Trends Microbiol. 2015;23:76-82. doi: 10.1016/j. tim.2014.10.010.

5. Oda Y, Hayashi F, Okada M. Longitudinal study of dental caries incidence associated with Streptococcus mutans and Streptococcus sobrinus in patients with intellectual disabilities. BMC Oral Health. 2015;15:102. doi: 10.1186/s12903-0150087-6.

6. Vos $T$, Flaxman AD, Naghavi M, Lozano R, Michaud C, Ezzati M, et al. Years lived with disability (YLDs) for 1160 sequelae of 289 diseases and injuries 1990-2010: a systematic analysis for the Global Burden of Disease Study 2010. Lancet. 2012;380:2163-96. doi: 10.1016/S0140-6736(12)61729-2.

7. WHO. International statistical classification of diseases and related health problems: World Health Organization; 2004. Available at: http://www.who.int/classifications/icd/en/. Accessed April 27, 2018.

8. Simmonds RS, Pearson L, Kennedy RC, Tagg JR. Mode of action of a lysostaphin-like bacteriolytic agent produced by Streptococcus zooepidemicus 4881. Appl Environ Microbiol. 1996;62:4536-41.

9. Simmonds RS, Simpson WJ, Tagg JR. Cloning and sequence analysis of zooA, a Streptococcus zooepidemicus gene encoding a bacteriocin-like inhibitory substance having a domain structure similar to that of lysostaphin. Gene. 1997;189:255-61. doi:10.1016/S0378-1119(96)00859-1.

10. Jain K. Studies on isolation and characterization of oral biofilm forming bacteria [dissertation]. Odisha, India: Department of Life Science; 2011.

11. O'Toole GA. Microtiter dish biofilm formation assay. JVis Exp. 2011:e2437. doi: 10.3791/2437.

12. Rubin BE, Sanders JG, Hampton-Marcell J, Owens SM, Gilbert JA, Moreau CS. DNA extraction protocols cause differences in 16S rRNA amplicon sequencing efficiency but not in community profile composition or structure. Microbiol Open. 2014;3:910-21. doi: 10.1002/mbo3.216.

13. Fási L, Wéber E, Czigle S, Sztojkov-Ivanov A, Liktor-Busa E, Blunden $\mathrm{G}$, et al. Isolation of iridoids and flavones from the anti-inflammatory, antioxidative and antimicrobial extract of Melampyrum barbatum. Planta Med. 2015;81:PW_60. doi: 10.1055/s-0035-1565684.

14. Chandrabhan D, Hemlata R, Renu B, Pradeep V. Isolation of dental caries bacteria from dental plaque and effect of tooth pastes on acidogenic bacteria. Open J Med Microbiol. 2012;2:65-9. doi: 10.4236/ojmm.2012.23009.

15. Kim YH, Lee SY. Comparison of three antibiotic susceptibility tests for viridans group streptococci. Inter J Oral Biol. 2011;36:163-6.

16. Aas JA, Paster BJ, Stokes LN, Olsen I, Dewhirst FE. Defining the normal bacterial flora of the oral cavity. J Clin Microbiol. 2005;43:5721-32. doi: 10.1128/JCM.43.11.5721-5732.2005.

17. Keijser BJ, Zaura E, Huse S, Van Der Vossen JM, Schuren $\mathrm{FH}$, et al. Pyrosequencing analysis of the oral microflora of healthy adults. I Dent Res. 2008;87:1016-20. doi: 10.1177/154405910808701104.

18. Kuramitsu HK, He X, Lux R, Anderson MH, Shi W. Interspecies interactions within oral microbial communities. Microbiol Mol Biol Rev. 2007;71:653-70. doi: 10.1128/MMBR.00024-07.

19. Kolenbrander PE, Palmer RJ Jr, Rickard AH, Jakubovics NS, Chalmers NI, Diaz PI. Bacterial interactions and successions during plaque development. Periodontol. 2000. 2006;42:4779. doi: 10.1111/j.1600-0757.2006.00187.x.

20. Lester K, Simmonds RS. Zoocin A and lauricidin in combination reduce Streptococcus mutans growth in a multispecies biofilm. Caries Res. 2012;46:185-93. doi: 10.1159/000337307.

21. Mcleod F, Simmonds R. Entry of antisense constructs into Streptococcus mutans. Clin Microbiol Infect. 2012;18:387.

22. Sergio A-O, Fabiola C-V, Guadalupe N-M, Blanca R-C, León $\mathrm{H}-\mathrm{O}$. Evaluation of antimycobacterium activity of the essential oils of cumin (Cuminum cyminum), clove (Eugenia caryophyllata), cinnamon (Cinnamomum verum), laurel (Laurus nobilis) and anis (Pimpinella anisum) against Mycobacterium tuberculosis. Adv Biol Chemist. 2013;3:4804. doi: 10.4236/abc.2013.35052.

23. Chaiya A, Saraya S, Chuakul W, Temsiririrkkul R. Screening for dental caries: Preventive activities of medicinal plants against Streptococcus mutans. Mahidol Univ J Pharmaceut Sci. 2013;40:9-17.

24. Alitonou GA, Tchobo FP, Avlessi F, Yehouenou B, Yedomonhan $\mathrm{P}$, Koudoro AY, et al. Chemical and biological investigations of Syzygium aromaticum L. essential oil from Benin. Int J Biolo Chem Sci. 2012;6:1360-7. doi: 10.4314/ijbcs.v6i3.37. 